\title{
INFLUENCE OF EVOLUTIONARY COMPUTING ON NUTRITION RECOMMENDATION: A SURVEY
}

\author{
${ }^{1}$ AlFayoumi, S.A., ${ }^{1}$ A.A. Hegazy and ${ }^{2}$ M.A. Belal \\ ${ }^{1}$ College of Computing and Information Technology, \\ Arab Academy for Science and Maritime Science Technology, Cairo, Egypt \\ ${ }^{2}$ College of Computers and Information, Helwan University, Cairo, Egypt
}

Received 2014-02-25; Revised 2014-03-25; Accepted 2014-05-08

\begin{abstract}
This study is a survey about how Evolutionary Computing doesn't play its important role in a vital field such as Nutrition. Evolutionary computing is a subset from the artificial intelligence umbrella that involves continuous optimization and combinational optimization which is based on searching methodologies. It has also a lot of algorithms that have played a main role in supporting the decision making and taking processes accurately and effectively. It is concerning many fields in our life such as Industry, Agriculture, Engineering, Transportation, Medicine and Nutrition, etc. One of these algorithms is Genetic Algorithm (GA) which is contributed to a lot of fields. Moreover, Nutrition is a wide field of research because it has several sides, medically, physically and psychologically and so on. But, has Genetic Algorithms been used to contribute to the field of nutrition? This survey illustrates that (GA) is not involved in nutrition computerized models or applications and it suggests building a model to promote a nutrition system using this powerful algorithm and this study presents a suggestion to build a model for nutrition as a future work that uses Genetic Algorithm.
\end{abstract}

Keywords: Evolutionary Computing, Evolutionary Algorithms (EA), Artificial Evolution (AE), Genetic Algorithms (GA), Nutrition

\section{INTRODUCTION}

Many problems nowadays can be solved in theory by intelligently searching through many possible solutions. An Evolutionary Algorithm (EA) is a subset of evolutionary computation, a generic populationsbased metaheuristic optimization algorithm and searching techniques. An EA uses mechanisms a inspired a by evolution, such as reproduction, mutation, recombination and selection. Candidate solutions to the optimization problem play the role of individuals in a population and the fitness function determines the quality of the solutions. Evolution of the population then takes place after the repeated application of the above operators. Artificial Evolution (AE) describes a process involving individual evolutionary algorithms;
EAs are individual components that participate in an AE. Evolutionary algorithms often perform well approximating solutions to all types of problems because they ideally do not make any assumption about the underlying fitness landscape. There are a lot of techniques that under the umbrella of EAs such as (http://en.wikipedia.org/wiki/Evolutionary_algrithms):

- Genetic Algorithms are a family of computational models inspired by evolution. These algorithms encode a potential solution to a specific problem on a simple chromosome like data structure and apply recombination operators to these structures so as to preserve critical information

- Ant colony optimization: Is based on the ideas of ant foraging by pheromone communication to form

Corresponding Author: AlFayoumi, S.A., College of Computing and Information Technology, Arab Academy for Science and Maritime Science Technology, Cairo, Egypt 
paths. Primarily suitedjfor combinatorial optimization and graph problems

- Bees algorithm: Is based on the foraging behavior of honey bees. It has been applied in many applications such as routing and scheduling

- Cuckoo search: Is inspired by the brooding parasitism of the cuckoo species. It also uses Lévy flights and thus it suits for global optimization problems

- Particle swarm optimization: Is based on the ideas of animal flocking behavior. Also primarily suited for numerical optimization problems

Such these techniques are based on Search methodology to find the fittest, most accurate solution for a lot of our vital problems then according to this solution; a correct decision must be taken. Evolutionary Algorithms can support fields like Medicine, Nutrition, Engineering, Agriculture and Transportation and so on. In next sections we will concentrate on Nutrition and its importance in enhancing the human's health and life generally and how EA can contribute to this problem (ATSMCD, 1995).

\section{NUTRITION}

Nutrition is a process, in order for an organism to sustain life, to obtain food with life supporting substances that can be digested and used by the organism. The better nutrition system, the better healthy life style is. Improving on the long term the dietary habits of people is a socially important task, which would help to enhance human's health. Nutrition has a lot of dimensions such as physical, psychological, social and medical and so on that must be put into consideration to determine the appropriate nutrition system. Figure 1 illustrates the factors that affect the nutrition process.

In the world of information technology there are applications were handled the issue of nutrition and how to recommend or promote the suitable nutrition system. For example in france 2007, Nutri-Educ is a nutrition software application developed in cooperation with the Diabetology Department of Toulouse's Rangueil University Hospital (Buisson, 2008). It aims at helping any person to balance their meals. Its main goal to enables the user to describe a meal and assess its content and in most cases to find a small set of acceptable actions which make it well-balanced and in accordance to the user's energetic needs. This software application is based on fuzzy arithmetic and heuristic search. Fuzzy arithmetic is used to perform computations on such quantities and fuzzy pattern matching provides measures. Fuzzy numbers are used to represent the inherent imprecision and fuzziness of food quantities and nutrient values as well as to model the gradual boundaries of the daily recommended values associated with each nutrient. Fuzzy arithmetic is used to perform computations on such quantities and fuzzy pattern matching provides measures of the compatibility of data to nutrient norms. Innovative visual gauges have been designed to display this information in a simple, yet comprehensive way. Finally, heuristic search algorithms are used to find a set of actions, acceptable from a nutritional point of view, which will transform the initial meal into a wellbalanced one. In the Nutri-Educ software application, there are 3 main parts can be seen.

\subsection{Meal Description}

It shows how a user chooses between several food pictures. The weight of each one is represented by a possibility distribution which was given by a dietician.

\subsection{Fuzzy Computation and Assessment}

It shows a meal which has been provided by the user and how it corresponds to the norms regarding four nutrients (energy, carbohydrates, fat, proteins). For example, the score $(7 / 10)$ is the combination of the compatibility measures associated with each nutrient, weighted by their importance.

\subsection{Meal Balancing}

The user may then press the 'balance' ('correction') button, which runs the meal balancing.

Also, in 2011, computer scientists in Taiwan constructed a web-based expert system for nutrition diagnosis by utilizing the expert system techniques in artificial intelligence Fig. 2 (Chen et al., 2012). The research implements Nutritional Care Process and Model (NCPM) defined by American Dietetic Association (ADA) in 2008 and integrate the nutrition diagnosis knowledge from dietetics professionals to establish the basics of building the rule based expert system with its knowledge base. The system is built using Microsoft Visual Studio 2008. NET framework 3.5SP1 utilizing the built in rule engine which comes with Windows Workflow Foundation. During nutrition diagnosis phase, a dietitian would identify and label the patient which indicates an occurrence, risk, or potential of developing some kind of nutritional issues that dietitians should treat. From the data gathering in the previous step, nutrition assessment, dietitians would organize and analyze the data and reveal diagnostic category and finally comes up with appropriate nutrition diagnosis. 


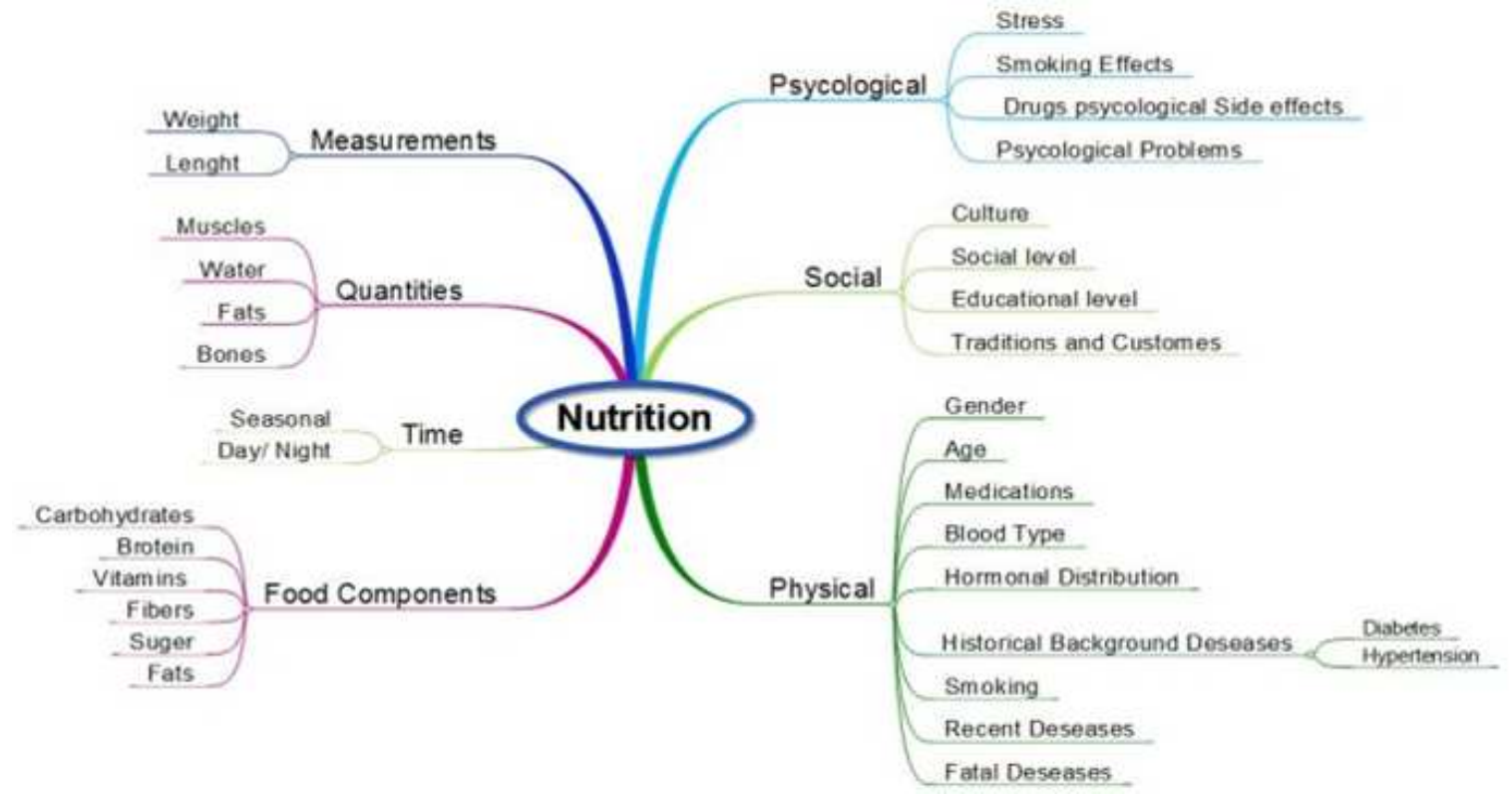

Fig. 1. Factors/dimensions of nutrition

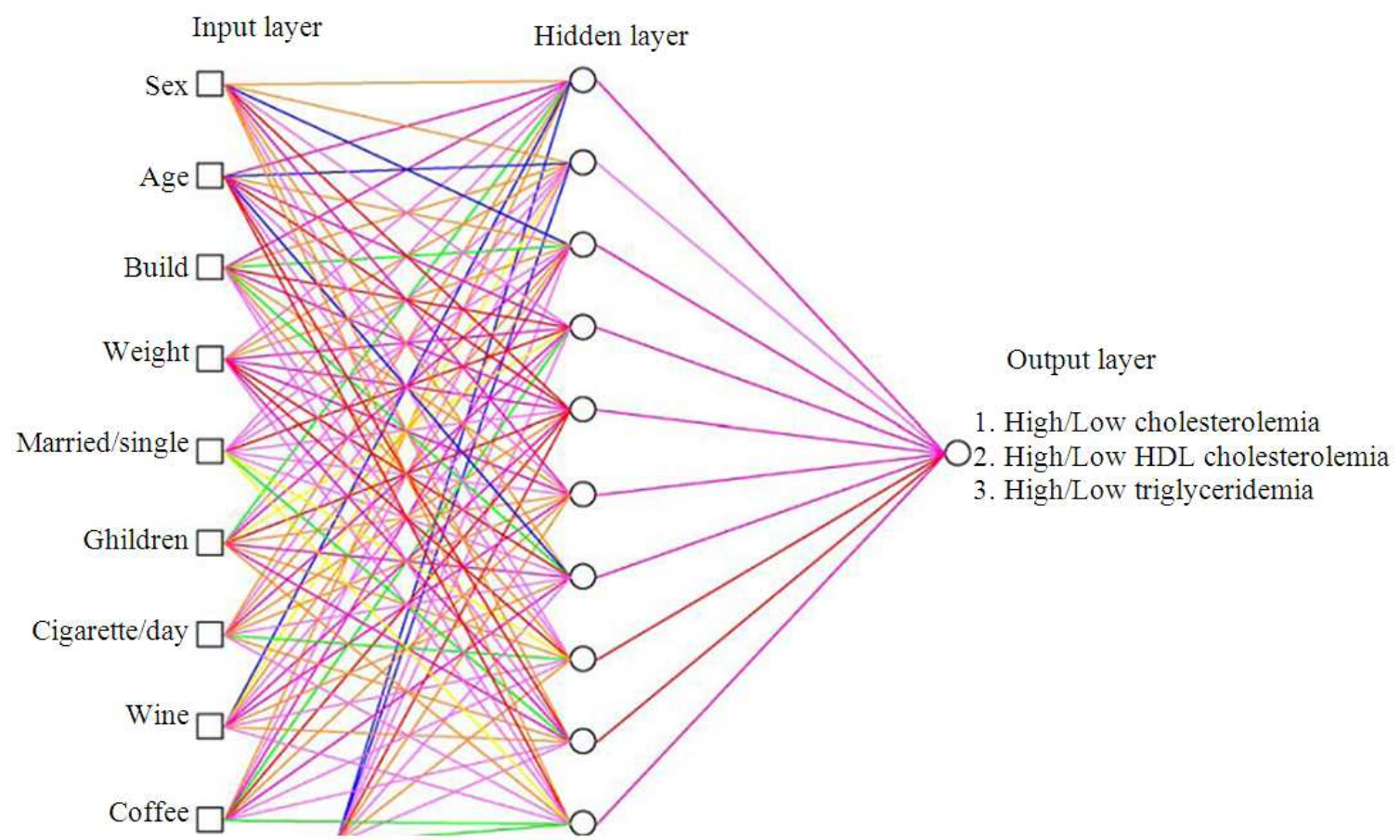

Fig. 2. Schematic drawing depicting the structure of the neural network developed. The input data are fed into the input layer of the net. Each node adjusts the weight for each of the variables it receives from the nodes until a value, which is a function of the input and corresponds to the correct answer, is generated in the output node. This is represented, in each network, by the existence of values exceeding or below the cutoffs for cholesterolemia, HDL cholesterol and triglyceridemia 
A nutrition diagnosis is different from a medical diagnosis which could be defined as a disease or some type of pathological disorder of a particular organ that can be treated. A nutrition diagnosis would change when patient's response changes. A medical diagnosis does not change as long as the disease condition stays. By analyzing the data gathered from nutritional assessment and come up with the correct nutrition diagnosis (es) presents dietitians with a way to set realistic and measurable goals, selecting fitting interventions and tracking improvement in achieving those expected goals.

A nutrition diagnosis contains three major components. They are problem (diagnostic label), etiology (cause/contributing factors) and signs/symptoms (defining characteristics). A diagnostic label is a phrase or sentence which describes the patient's condition such as increased/decreased, or having risk of. In etiology, we state the cause of the problem. This helps in determining whether or not we should be expecting that the following nutritional intervention would effectively help the patient with his/her nutritional condition. By recording the etiology, we may also gain the benefit of identifying the cause and thus correct it. In the signs and symptom components of the nutritional diagnosis, we record both subjective and objective signs and symptoms for the patient. The signs and symptoms component includes patient's biochemical data, anthropometric measurements, physical examination findings and food/nutrition history. These data allows quantifying the problem and permit us to evaluate and give a severity ranking. The symptoms are changes the patient verbally tells the dietitians while the signs are observable fluctuations in the patient's health status.

While in 2012, in USA, researchers proposed a proofof-concept machine-learning expert system that learned knowledge of lifestyle specially nutrition system and the associated 10-year Cardiovascular Disease (CVD) risks from individual-level data. The expert system prioritizes lifestyle options and identifies the one that maximally reduce an individual's 10-year CVD risk by (1) using the knowledge learned from the data historical and (2) communicating for patient-specific cardiovascular risk information and personal limitations and preferences (as defined by variables used in this study). As a result, the optimal lifestyle is not only prioritized based on an individual's characteristics but is also relevant to personal circumstances. They also explored probable uses and tested the system in several examples using real-world scenarios and patient preferences. For example, the system identifies the most effective lifestyle activities as the starting point for an individual's behavior change, shows different levels of Body Mass Index (BMI) changes and the associated CVD risk reductions to encourage weight loss, identifies whether weight loss or smoking cessation is the most urgent change for a diabetes patient. Answers to the questions noted above vary based on an individual's characteristics. Their validation results from clinical trial simulations, which compared original with the optimal lifestyle using an independent dataset, show that the optimal individualized patient centered lifestyle consistently, reduced 10-year CVD risks. They used the prediction and optimization based decision support system algorithm PODSS to build this expert system. The PODSS algorithm allows the choice of any classification method that is deemed appropriate for a particular problem. They chose k-NN as their classifier for both conceptual and clinical reasons. Conceptually, CVD risks were computed from people with similar characteristics. This transparency allows them to easily visualize and understand how CVD risks were computed and why the system concluded that a particular change would result in a risk reduction. Clinically, due to the nature of data storage for prediction, a patient could visualize people's (de-identified) lifestyle and outcomes. They expected such a visualization mechanism would enable observational learning and further help healthy lifestyle adoption. In fact, in social cognitive theory, observing other people's behavior is one important element in self-efficacy that improves an individual's confidence in successfully carrying out a behavior.

In Institution of Information systems in France, 2000, To improve someone's diet, the educational dimension is as important as the arithmetic consideration of nutrients, because people rely mostly on habits gained in the family kitchen and at the dining table. This book discusses issues about knowledge-based tutoring systems acting on problem solving domains. The Nutri-Expert (IRIT, 2000) system is then presented in detail: It uses possibility theory to take the inherent imprecision of the database numbers and the input data into account properly and introduces the educational aspects via the daily use of an algorithm that finds a minimal transformation of a given meal to make it well balanced. This algorithm is based on a heuristic search in a state space of hypothetical meals. Different versions have been developed and evaluated using a test database of real meals. Nutri-Expert is widely used by our patients at home and several medical vaHdations have measured its biological and educational benefits. Nutri-Expert is composed of several closely linked modules. The main 
one is the analysis module, which will be described in detail in this section and which allows patients to analyze particular meals and get them well balanced. A second module gives different kinds of well balanced daily diets (ordinary, green, exotic, parties). The quantities of foods are adapted to the patients' caloric needs and their medical problems and a proposed meal can be downloaded to the analysis module to be modified and corrected. There are more than 200 such meals in the database. A third module acts as an encyclopedia on nutrition, giving information and definitions about the words and concepts used in the software (calories, fats, proteins, blood glucose, criteria of equilibrium,). It also gives complete recipes (more than 60) for dishes used in the other two modules.

\section{GENETIC ALGORITHM}

Furthermore, as far as a process of search, EA do not play a real role in solving the problem of promoting the suitable nutrition system. One of EA can be powerful and give fittest solutions in such this problem is Genetic Algorithm (GA). Genetic algorithms as shown in Fig. $\mathbf{3}$ are search Algorithms based on the mechanisms of natural selection and genetics. They have been applied to search, optimization and machine learning problems with great success.

Let's show the contributions of GA in building models in medical fields. These models were made to optimize some problems like treatment scheduling, misclassification and prediction. In 2010, genetic algorithm was used in optimizing a treatment scheduling problem by some academics in UK (Petrovic et al., 2011). They presented a multi-objective optimization model and algorithms for scheduling of radiotherapy treatments for categorized cancer patients. The model is developed considering real life radiotherapy treatment processes at Arden Cancer Centre, in the UK. The scheduling model considered various real life constraints, such as doctors' rota, machine availability, patient's category, waiting time targets (i.e., the time when a patient should receive the first treatment fraction) and so on. Two objectives were defined: Minimization of the Average patient's waiting time and minimization of Average length of breaches of waiting time targets. Three Genetic Algorithms (GAs) were developed and implemented which treat radiotherapy patient categories, namely emergency, palliative and radical patients in different ways: (1) Standard- GA, which considers all patient categories equally, (2) KB-GA, which has an embedded knowledge on the scheduling of emergency patient category and (3) Weighted-GA, which operates with different weights given to the patient categories. The performance of schedules generated by using the three GAs is compared using the statistical analyses. The results show that KB-GA generated the schedules with best performance considering emergency patients and slightly outperforms the other two GAs when all patient categories are considered simultaneously. KB-GA and Weighted-GA generated better performance schedules for emergency and palliative patients than Standard-GA. But, In 2011, group of researchers in Korea had proposed a new CBR method called Cost-Sensitive Case-Based Reasoning (CSCBR) that can incorporate unequal misclassification costs into $\mathrm{CBR}$ and optimize the number of neighbors dynamically using a genetic algorithm (Parka et al., 2011). Their research studied the new learning technique called Cost-Sensitive CaseBased Reasoning (CSCBR) incorporating unequal misclassification cost into CBR model. Conventional CBR is now considered as a suitable technique for diagnosis, prognosis and prescription in medicine. However it lacks the ability to reflect asymmetric misclassification and often assumes that the cost of a positive diagnosis (an illness) as a negative one (no illness) is the same with that of the opposite situation. Thus, the objective of this research is to overcome the limitation of conventional CBR and encourage applying CBR to many real world medical cases associated with costs of asymmetric misclassification errors. The main idea involves adjusting the optimal cut-off classification point for classifying the absence or presence of diseases and the cut-off distance point for selecting optimal neighbors within search spaces based on similarity distribution. These steps are dynamically adapted to new target cases using a genetic algorithm. They applied this proposed method to five real medical datasets and compare the results with two other cost-sensitive learning methods-C5.0 and CART. As a result, they found that the total misclassification cost of CSCBR is lower than other cost-sensitive methods in many cases. Even though the genetic algorithm has limitations in terms of unstable results and over-fitting training data, CSCBR results with GA are better overall than those of other methods. Also the paired t-test results indicate that the total misclassification cost of CSCBR is significantly less than C5.0 and CART for several datasets. While, in machine learning and knowledge discovery field and away from the medical field, rule extraction and prediction problem was enhanced by using GA in 2012 in India (Sarkara, 2012). 


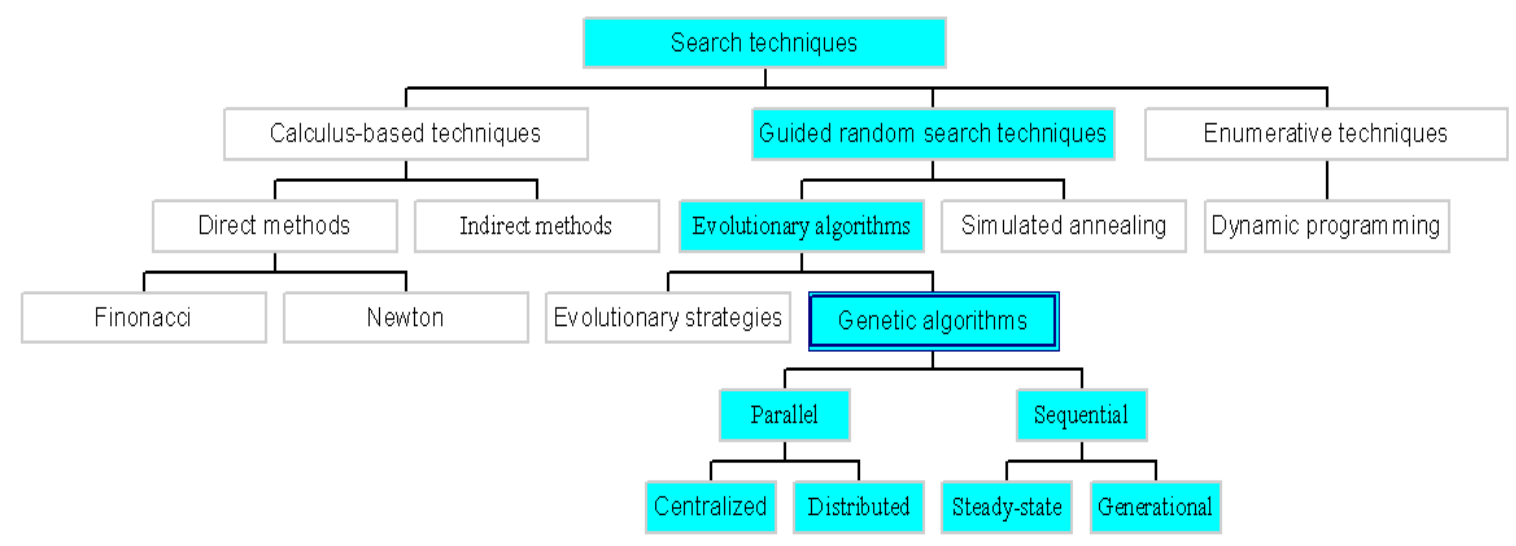

Fig. 3. Genetic Algorithm as a search technique

Researchers introduced an accuracy-based learning system Called Decision Tree and Genetic Algorithm (DTGA) that aims to improve prediction accuracy over any classification problem irrespective to domain, size, dimensionality and class distribution. More specifically, the proposed system consists of two rule inducing phases. In the first phase, a base classifier, C4.5 (a decision tree based rule inducer) was used to produce rules from training data set, whereas Genetic Algorithm (GA) in the next phase refined them with the aim to provide more accurate and high-performance rules for prediction. The system had been compared with competent non-GA based systems: Neural network, Naïve Bayes, rule-based classifier using rough set theory and C4.5 (i.e., the base classifier of DTGA), on a number of benchmark datasets collected from University of California at Irvine (UCI) machine learning repository. Empirical results demonstrate that the proposed hybrid approach provides marked improvement in a number of cases.

\section{THE PROPOSED MODEL}

As shown above in nutrition section, a lot of nutrition models were made by using Neural Networks, Experts systems, fuzzy logic and heuristic search. No applications, systems or models which applied by EAs were using genetic algorithms. The intended model is how to recommend or promote a suitable nutrition supplementary program for a person according to his measurement, either medical or personal measurements such as age, gender, weight, height, type of work, blood sugar, blood pressure, cholesterol, uric acid and hemoglobin and so on using genetic algorithm.
The output will be some recommendations that enhanced the healthy life style for each person such as how much sugar and salt should take daily, how many glasses of water must drink, what are vitamins must be taken, the quantity of protein in form of red meat, white meat or vegetarian protein, how many minutes should assign daily for exercise and how much fats and carbohydrates are needed and so on.

\section{CONCLUSION}

After illustrating the evolutionary computing and its importance, nutrition models and their techniques used in, such as fuzzy logic, heuristic search, expert systems and neural networks, genetic algorithms and fields supported by it, it is apparent that, so far, there is no nutrition model uses (GA) in its implementation despite it could lead to promising results if it is used. The motivation here, is to apply genetic algorithm on the intended model and show if the results that will be obtained from this model will be better or the previous mentioned models and techniques are better.

\section{REFERENCES}

ATSMCD, 1995. Genetic algorithms: A tutorial salvatore mangano computer design.

Buisson, J.C., 2008. Nutri-Educ, a nutrition software application for balancing meals, using fuzzy arithmetic and heuristic search algorithms. Artificial Intell. Med., 42: 213-227.

Chen, Y., C.Y. Hsu, L. Liu and S. Yang, 2012. Constructing a nutrition diagnosis expert system. Expert Syst. Applic., 39: 2132-2156. DOI: 10.1016/j.eswa.2011.07.069 
IRIT, 2000. Knowledge development expert systems and theirapplication in nutrition jean-christophe buisson. France.

Parka, Y.J., S.H. Chuna and B.C. Kim, 2011. Costsensitive case-based reasoning using a genetic algorithm: Application to medical diagnosis. Artifi. Intell. Med., 51: 133-145. DOI: 10.1016/j.artmed.2010.12.001
Petrovic, D., M. Morshed and S. Petrovic, 2011. Multiobjective genetic algorithms for scheduling of radiotherapy treatments for categorised cancer patients. Expert Syst. Applic., 38: 6994-7002. DOI: 10.1016/j.eswa.2010.12.015

Sarkara, B.K., 2012. Shib sankar sanab, kripasindhu chaudhuric. A genetic algorithm-based rule extraction system. India. 